\title{
VARIATIONAL ESTIMATES FOR DISCRETE SCHRÖDINGER OPERATORS WITH POTENTIALS OF INDEFINITE SIGN
}

\author{
D. DAMANIK ${ }^{1,3}$, D. HUNDERTMARK ${ }^{2}$, R. KILLIP ${ }^{1}$, AND B. SIMON ${ }^{1,4}$
}

\begin{abstract}
Let $H$ be a one-dimensional discrete Schrödinger operator. We prove that if $\sigma_{\text {ess }}(H) \subset[-2,2]$, then $H-H_{0}$ is compact and $\sigma_{\text {ess }}(H)=[-2,2]$. We also prove that if $H_{0}+\frac{1}{4} V^{2}$ has at least one bound state, then the same is true for $H_{0}+V$. Further, if $H_{0}+\frac{1}{4} V^{2}$ has infinitely many bound states, then so does $H_{0}+V$. Consequences include the fact that for decaying potential $V$ with $\liminf _{|n| \rightarrow \infty}|n V(n)|>1, H_{0}+V$ has infinitely many bound states; the signs of $V$ are irrelevant. Higher-dimensional analogues are also discussed.
\end{abstract}

\section{INTRODUCTION}

Let $H$ be a Schrödinger operator on $\ell^{2}(\mathbb{Z})$,

$$
(H u)(n)=u(n+1)+u(n-1)+V(n) u(n)
$$

with bounded potential $V: \mathbb{Z} \rightarrow \mathbb{R}$. The free Schrödinger operator, $H_{0}$, corresponds to the case $V=0$. One of our main results in this paper is

Theorem 1. If $\sigma_{\text {ess }}(H) \subset[-2,2]$, then $V(n) \rightarrow 0$ as $|n| \rightarrow \infty$, that is, $H-H_{0}$ is compact.

Remark. By Weyl's Theorem, we have the immediate corollary that $\sigma_{\text {ess }}(H)=$ $[-2,2]$ if and only if $V(n) \rightarrow 0$.

Our motivation for this result came from two sources:

Theorem 2 (Killip-Simon [7]). If $\sigma(H) \subset[-2,2]$, then $V=0$.

Theorem 3 (Rakhmanov [12]; see also Denisov [5], Nevai [1], and references therein). Let $J$ be a general half-line Jacobi matrix on $\ell^{2}\left(\mathbb{Z}^{+}\right)$,

$$
(J u)(n)=a_{n} u(n+1)+b_{n} u(n)+a_{n-1} u(n-1)
$$

where $a_{n}>0$ and $\mathbb{Z}_{+}=\{1,2, \ldots\}$. Suppose that $[-2,2]$ is the essential support of the a.c. part of the spectral measure and also the essential spectrum. Then $\lim _{n \rightarrow \infty}\left|a_{n}-1\right|+\left|b_{n}\right|=0$, that is, $J$ is a compact perturbation of $J_{0}$, the Jacobi matrix with $a_{n} \equiv 1, b_{n} \equiv 0$.

Date: November 5, 2002.

1 Mathematics 253-37, California Institute of Technology, Pasadena, CA 91125. E-mail: damanik@its.caltech.edu; killip@its.caltech.edu; bsimon@caltech.edu.

${ }^{2}$ Institut Mittag-Leffler, Auravägen 17, S-182 60 Djursholm, Sweden. On leave from Department of Mathematics, University of Illinois at Urbana-Champaign, 1409 W. Green Street, Urbana, IL 61801-2975.

3 Supported in part by NSF grant DMS-0227289.

4 Supported in part by NSF grant DMS-0140592. 
While Theorem 3 motivated our thoughts, it is not closely related to the result. Not only are the methods different, but it holds for any a priori $a_{n}$; whereas our results require some a priori estimates like $a_{n} \rightarrow 1$ as $|n| \rightarrow \infty$. For example, if $a_{n} \equiv \frac{1}{2}$ and $b_{n}$ takes values +1 and -1 over longer and longer intervals, it is not hard to see that $\sigma(J)=[-2,2]$, but clearly, $J-J_{0}$ is not compact. Thus Theorem 1 , unlike Theorem 3, is essentially restricted to discrete Schrödinger operators.

For continuum Schrödinger operators, consideration of sparse positive nondecaying potentials shows that $\sigma(H)=[0, \infty)$ is possible even when $(H+1)^{-1}-\left(H_{0}+1\right)^{-1}$ is not compact. The reason is that our proof depends essentially - as does Theorem 2 - on the fact that $\sigma(H)$ has two sides in the discrete case.

Theorem 1 has an interesting corollary:

Corollary 4. Let $H$ be an arbitrary one-dimensional discrete Schrödinger operator. Then $\sup \sigma_{\mathrm{ess}}(H)-\inf \sigma_{\mathrm{ess}}(H) \geq 4$ with equality if and only if $V(n) \rightarrow V_{\infty} a$ constant as $|n| \rightarrow \infty$.

Proof. Let $a_{+}=\sup \sigma_{\text {ess }}(H), a_{-}=\inf \sigma_{\text {ess }}(H)$. If $a_{+}-a_{-} \leq 4$, then $H-\frac{1}{2}\left(a_{+}+a_{-}\right)$ is a Schrödinger operator with essential spectrum in $[-2,2]$. So Theorem 1 implies the original $V(n) \rightarrow \frac{1}{2}\left(a_{+}+a_{-}\right)$. Hence, $a_{+}-a_{-}=4$ and $\sigma_{\mathrm{ess}}=\left[a_{-}, a_{+}\right]$.

Remarks. (a) A similar argument combined with Theorem 2 implies that if $\sup \sigma(H)-\inf \sigma(H) \leq 4$, then $V$ is a constant.

(b) If $V(n)=(-1)^{n} \lambda$ and $\lambda$ is large, standard Floquet theorem arguments show that $\sigma(H)$ has two bands centered about $\pm\left(\lambda+O\left(\frac{1}{\lambda}\right)\right)$ and of width $O\left(\frac{1}{\lambda}\right)$. Thus, while the size of the convex hull of $\sigma(H)$ is of size at least 4 , the size of $\sigma(H)$ can be arbitrarily small. Indeed, by results of Deift-Simon [4], if $H$ has purely a.c. spectrum, (e.g., $V$ periodic), the total size of $\sigma(H)$ is at most 4 .

While Theorem 1 is our main motivating result, the ideas behind it yield many other results about the absence of eigenvalues and about the finiteness or infinitude of their number for Schrödinger operators not only on the line, but also on the half-line or in higher dimensions. Included in our results are

(i) Theorem 1 holds in two dimensions and is false in three or more dimensions (see Theorems 4.1 and 4.2). This is connected to the fact that Schrödinger operators in one and two dimensions always have a bound state for nontrivial attractive potentials (see [9, pp. 156-157] and [8, 15]), whereas in three and more dimensions, small attractive potentials need not have bound states by the Cwikel-Lieb-Rozenblum bound [1, 10, 14].

(ii) For a half-line discrete Schrödinger operator, $H$, if $\sigma(H)=[-2,2]$ (i.e., no bound states), then (see Theorem 5.2

$$
|V(n)| \leq 2 n^{-1 / 2}
$$

On the other hand (see Theorem 5.2), there are examples, $V_{k}(n)$, with no bound states and $\lim _{k} \sup _{n} n^{1 / 2}\left|V_{k}(n)\right|=1$. This shows that the power $\frac{1}{2}$ in (1.3) cannot be made larger. It also shows that the constant, 2, cannot be made smaller than 1 . (The optimal constant is $\sqrt{2}$. This is proved in [3].)

(iii) The examples in (ii) are necessarily sparse in that if $|V(n)| \geq \mathrm{Cn}^{-\alpha}$ and $H$ has only finitely many bound states, then $\alpha \geq 1$. Indeed, we will prove (see Theorem 5.6) that if $\alpha=1$ and $C>1$ or $\alpha<1$ and $C>0$, then $H$ has an infinity of bound states. This will follow from the very general theorem: 
Theorem 5. Let $V(n) \rightarrow 0$. If $H_{0}+\frac{1}{4} V^{2}$ has at least one (resp., infinitely many) eigenvalues outside $[-2,2]$, then $H_{0}+V$ has at least one (resp., infinitely many) eigenvalues outside $[-2,2]$.

Theorem 3.1 extends this result to all dimensions.

(iv) If $|V(n)| \geq C n^{-\alpha}$ and $\alpha<1$, we will prove suitable eigenvalue moments diverge.

The starting point of the present paper is the discussion at the end of Section 10 of [7] that it should be possible to prove Theorem 2 variationally with suitable second-order perturbation trial functions. Second-order eigenvalue perturbation theory has a change of the first-order eigenfunction by a term proportional to $V$. Thus, our variational trial function will have two pieces: $\varphi$ and an extra piece, proportional to $V \varphi$.

The second key idea is to make use of the fact that the spectrum of $H_{0}$ has two sides, and we can use a pair of trial functions: one to get an eigenvalue below -2 and one to get an eigenvalue above +2 . By combining them, we will have various cancellations that involve terms whose sign is uncertain. Explicitly, given a pair of trial vectors $\varphi_{+}$and $\varphi_{-}$, we define

$$
\Delta\left(\varphi_{+}, \varphi_{-} ; V\right)=\left\langle\varphi_{+},(H-2) \varphi_{+}\right\rangle+\left\langle\varphi_{-},(-H-2) \varphi_{-}\right\rangle
$$

where $H$ is given by (1.1). If $\Delta>0$, either $\left\langle\varphi_{+},(H-2) \varphi_{+}\right\rangle>0$ or $\left\langle\varphi_{-},(H+\right.$ $\left.2) \varphi_{-}\right\rangle<0$, that is, there is either an eigenvalue above 2 or below -2 !

In choosing $\varphi_{-}$relative to $\varphi_{+}$, it will help to use the unitary operator $U$ on $\ell^{2}(\mathbb{Z})$ given by

$$
(U \varphi)(n)=(-1)^{n} \varphi(n)
$$

so that

$$
U H_{0} U^{-1}=-H_{0} \quad U V U^{-1}=V
$$

The key calculation in Section 2 will be that

$$
\Delta\left(\varphi+\frac{1}{4} V \varphi, U\left(\varphi-\frac{1}{4} V \varphi\right)\right) \geq 2\left\langle\varphi,\left[H_{0}+\frac{1}{4} V^{2}-2\right] \varphi\right\rangle
$$

For example, this immediately implies the "at least one bound state" part of Theorem 5. If $H_{0}+\frac{1}{4} V^{2}$ has a bound state, $\varphi$, we must have $\left.\left\langle\varphi,\left(H_{0}+\frac{1}{4} V^{2}\right) \varphi\right\rangle\right\rangle$ $2\langle\varphi, \varphi\rangle$, so $\Delta>0$.

The current paper complements [2]. That paper provided upper bounds on the distance from $[-2,2]$ of eigenvalues of discrete Schrödinger operators with oscillatory potentials. This paper provides lower bounds. In particular, there it was shown the Jacobi matrix with $a_{n} \equiv 1, b_{n}=\frac{\beta(-1)^{n}}{n}$ has finitely many eigenvalues if $|\beta| \leq \frac{1}{2}$. Here, we prove infinitely many (see Theorem 5.7) if $|\beta|>1$. We also show, by ad hoc methods, that there are no eigenvalues for $|\beta| \leq 1$ (see Proposition 5.9).

In Section 2, we prove variational estimates, including (1.7). In Section 3, we prove Theorem 5. In Section 1 , we prove Theorem 1 and provide a new proof of Theorem 2. Sections 2 (4) also discuss higher dimensions. In Section 5, we study the one-dimensional situation more closely.

We thank Andrej Zlatoš for useful discussions. 


\section{VARiational Estimates}

On $\ell^{2}\left(\mathbb{Z}^{\nu}\right)$, define $H_{0}$ by

$$
\left(H_{0} u\right)(n)=\sum_{|j|=1} u(n+j)
$$

so

$$
-2 \nu \leq H_{0} \leq 2 \nu
$$

For $V$, a bounded function on $\mathbb{Z}^{\nu}$, let

$$
H=H_{0}+V
$$

We are interested in the spectrum of $H$ outside $[-2 \nu, 2 \nu]=\sigma\left(H_{0}\right)$.

If we define $U$ on $\ell^{2}\left(\mathbb{Z}^{\nu}\right)$ by

$$
(U \varphi)(n)=(-1)^{|n|} \varphi(n)
$$

where $|n|=\left|n_{1}\right|+\cdots+\left|n_{\nu}\right|$, then

$$
U H_{0} U^{-1}=-H_{0} \quad U V U^{-1}=V
$$

We define, for $\varphi_{+}, \varphi_{-} \in \ell^{2}\left(\mathbb{Z}^{\nu}\right)$,

$$
\Delta\left(\varphi_{+}, \varphi_{-} ; V\right)=\left\langle\varphi_{+},(H-2 \nu) \varphi_{+}\right\rangle+\left\langle\varphi_{-},(-H-2 \nu) \varphi_{-}\right\rangle
$$

$\Delta>0$ implies that $H$ has spectrum outside $[-2 \nu, 2 \nu]$ and, as we will see, $\Delta\left(\varphi_{+}^{(n)}, \varphi_{-}^{(n)} ; V\right)>0$ for suitable $\varphi_{ \pm}^{(n)}$ implies the spectral projection $\chi_{\mathbb{R} \backslash[-2 \nu, 2 \nu]}(H)$ has infinite dimension.

Note first that

Proposition 2.1. If $f, g \in \ell^{2}\left(\mathbb{Z}^{\nu}\right)$, then

$$
\Delta(f+g, U(f-g) ; V) \geq 2\left\langle f,\left(H_{0}-2 \nu\right) f\right\rangle-8 \nu\|g\|^{2}+4 \operatorname{Re}\langle f, V g\rangle
$$

Proof. By (2.5),

$$
\begin{aligned}
\Delta(f+g, U(f-g) ; V)=\langle( & \left.f+g),\left(H_{0}-2 \nu+V\right)(f+g)\right\rangle \\
& +\left\langle(f-g),\left(H_{0}-2 \nu-V\right)(f-g)\right\rangle \\
= & 2\left\langle f,\left(H_{0}-2 \nu\right) f\right\rangle+2\left\langle g,\left(H_{0}-2 \nu\right) g\right\rangle+4 \operatorname{Re}\langle f, V g\rangle
\end{aligned}
$$

By (2.2), $H_{0} \geq-2 \nu$, so

$$
\left\langle g,\left(H_{0}-2 \nu\right) g\right\rangle \geq-4 \nu\|g\|^{2}
$$

This yields (2.7).

One obvious choice is to take $f=\varphi, g=\gamma V \varphi$. The $V$-terms on the right side of (2.7) are then

$$
\|V \varphi\|^{2}\left(-8 \nu \gamma^{2}+4 \gamma\right)
$$

which is maximized at $\gamma=\frac{1}{4 \nu}$, where $-8 \nu \gamma^{2}+4 \gamma=\frac{1}{2 \nu}$. Thus we have a generalization of (1.7).

Theorem 2.2. For any $\varphi \in \ell^{2}\left(\mathbb{Z}^{\nu}\right)$,

$$
\Delta\left(\left(1+\frac{1}{4 \nu} V\right) \varphi, U\left(1-\frac{1}{4 \nu} V\right) \varphi ; V\right) \geq 2\left\langle\varphi,\left(H_{0}-2 \nu+\frac{1}{4 \nu} V^{2}\right) \varphi\right\rangle
$$

In some applications, we will want to be able to estimate $\|f \pm g\|$ in terms of $f$, and so want to cut off $V g$. We have 
Theorem 2.3. For any $F \in \ell^{\infty}$ with $0 \leq F \leq 1$, we have

$$
\Delta\left(\varphi\left(1+(4 \nu)^{-1} F V\right), U \varphi\left(1-(4 \nu)^{-1} F V\right) ; V\right) \geq 2\left\langle\varphi,\left(H_{0}-2 \nu+(4 \nu)^{-1} F V^{2}\right) \varphi\right\rangle
$$

Proof. By taking $g=\gamma F V \varphi, f=\varphi$, the $V$-terms in (2.7) are

$$
-8 \nu \gamma^{2}\|F V \varphi\|^{2}+4 \gamma\left\langle V \varphi, F V \varphi_{-}\right\rangle
$$

in place of (2.8). Since $0 \leq F \leq 1$, we have $-F^{2} \geq-F$, so

$$
-\|F V \varphi\|^{2} \geq-\langle V \varphi, F V \varphi\rangle
$$

and (2.10) results.

The properties of $H_{0}$ needed above are only (2.2) and (2.5). If $J$ is a Jacobi matrix (1.2) and $J_{1}$ is the Jacobi matrix with the same values of $a_{n}$ but with $b_{n}=0$, then $U J_{1} U^{-1}=-J_{1}$. (2.2) is replaced by

$$
J_{1} \geq-\alpha
$$

where

One has

$$
\alpha=\max _{n}\left(a_{n}+a_{n+1}\right)
$$

Theorem 2.4. For any $\varphi \in \ell^{2}\left(\mathbb{Z}_{+}\right)$, with $\varphi_{ \pm}=(1 \pm \gamma V) \varphi\left(\right.$ where $\left.\gamma=(2+\alpha)^{-1}\right)$, we have

$$
\left\langle\varphi_{+},(J-2) \varphi_{+}\right\rangle+\left\langle U \varphi_{-},(-2-J) U \varphi_{-}\right\rangle \geq 2\left\langle\varphi,\left(J_{1}-2+\gamma b^{2}\right) \varphi\right\rangle
$$

\section{A $V^{2}$ Comparison Theorem}

Our goal in this section is to prove the following extension of Theorem 5:

Theorem 3.1. Let $V$ be defined on $\mathbb{Z}^{\nu}$. Let $V(n) \rightarrow 0$ as $|n| \rightarrow \infty$. If $H_{0}+$ $(4 \nu)^{-1} V^{2}$ has at least one eigenvalue (resp., infinitely many) outside $[-2 \nu, 2 \nu]$, then so does $H_{0}+V$.

The key to this will be Theorem 2.2, but we will also need

Lemma 3.2. Let $W \geq 0$ on $\mathbb{Z}^{\nu}$ with $W(n) \rightarrow 0$ as $|n| \rightarrow \infty$. If $H_{0}+W$ has infinitely many eigenvalues in $(2 \nu, \infty)$, then we can find $\left\{\varphi_{n}\right\}_{n=1}^{\infty}$ with $\left\langle\varphi_{n},\left(H_{0}+\right.\right.$ $\left.W) \varphi_{n}\right\rangle>2 \nu\left\|\varphi_{n}\right\|^{2}$, so that each $\varphi_{n}$ has finite support and

$$
\operatorname{dist}\left(\operatorname{supp}\left(\varphi_{n}\right), \operatorname{supp}\left(\varphi_{m}\right)\right) \geq 2
$$

for all $n \neq m$.

Proof. Let $\Lambda_{k}=\left\{n \in \mathbb{Z}^{\nu}\left|\max _{i=1, \ldots, \nu}\right| n_{i} \mid \leq k\right\}$. We first claim that for every $k$, there exists $\psi$ with $\psi=0$ on $\Lambda_{k}$ so that $\left\langle\psi,\left(H_{0}+W\right) \psi\right\rangle>2 \nu\|\psi\|^{2}$. For let $\widetilde{H}_{0}$ be $H_{0}$ with Dirichlet boundary conditions on $\partial \Lambda_{k}$, that is, dropping off-diagonal terms $H_{0, i j}$ with $i \in \Lambda_{k}, j \notin \Lambda_{k}$ or vice-versa. $\widetilde{H}_{0}-H_{0}$ is finite rank, so $\widetilde{H}_{0}+W$ has infinitely many eigenvalues in $(2 \nu, \infty)$. But $\widetilde{H}_{0}+W$ is a direct sum of an operator on $\ell^{2}\left(\Lambda_{k}\right)$ and one on $\ell^{2}\left(\mathbb{Z}^{\nu} \backslash \Lambda_{k}\right)$. Since $\operatorname{dim} \ell^{2}\left(\Lambda_{k}\right)<\infty$, we can find $\psi \in \ell^{2}\left(\mathbb{Z}^{\nu} \backslash \Lambda_{k}\right)$ so $\left\langle\psi,\left(H_{0}+W\right) \psi\right\rangle=\left\langle\psi,\left(\widetilde{H}_{0}+W\right) \psi\right\rangle>2 \nu\|\psi\|^{2}$.

Now pick $\varphi_{n}$ inductively as follows. After picking $\left\{\varphi_{n}\right\}_{n=1}^{N}$, we have each $\varphi_{n}$ has finite support, so there is a $\Lambda_{k}$ with each $\varphi_{n}=0$ on $\mathbb{Z}^{\nu} \backslash \Lambda_{k}, n=1, \ldots, N$. 
By the initial argument, pick $\psi_{N+1}$ vanishing on $\Lambda_{k+1}$ so that $\left\langle\psi_{N+1},\left(H_{0}+\right.\right.$ $\left.W) \psi_{N+1}\right\rangle>2 \nu\left\langle\psi_{N+1}, \psi_{N+1}\right\rangle$ and $\psi_{N+1}=0$ on $\Lambda_{k+1}$. Let $\psi_{N+1}^{(m)}$ be finitely supported approximations to $\psi_{N+1}$ which vanish on $\Lambda_{k+1}$. By continuity, for some $m$, $\left\langle\psi_{N+1}^{(m)},\left(H_{0}+W\right) \psi_{N+1}^{(m)}\right\rangle>2 \nu\left\langle\psi_{N+1}^{(m)}, \psi_{N+1}^{(m)}\right\rangle$. Pick $\varphi_{N+1}=\psi_{N+1}^{(m)}$.

Proof of Theorem 3.1. If $H_{0}+(4 \nu)^{-1} V^{2}$ has at least one eigenvalue outside $[-2 \nu, 2 \nu]$, there exists $\varphi$ with $\left\langle\varphi,\left(H_{0}+\frac{1}{4 \nu} V^{2}-2 \nu\right) \varphi\right\rangle>0$. By (2.9), $H_{0}+V$ has some eigenvalue outside $[-2 \nu, 2 \nu]$.

If $H_{0}+(4 \nu)^{-1} V^{2}$ has infinitely many eigenvalues, by Lemma 3.2, there exist $\varphi_{n}$ obeying (3.1) so that $\left\langle\varphi_{n},\left(H_{0}+\frac{1}{4} V^{2}\right) \varphi_{n}\right\rangle>2 \nu\left\|\varphi_{n}\right\|^{2}$. By (2.9), we can find $\psi_{n}$ with either $\left\langle\psi_{n},\left(H_{0}+V\right) \psi_{n}\right\rangle>2 \nu\left\|\psi_{n}\right\|^{2}$ or $\left\langle\psi_{n},\left(H_{0}+V\right) \psi_{n}\right\rangle<-2 \nu\left\|\psi_{n}\right\|^{2}$ and $\operatorname{supp}\left(\psi_{n}\right) \subset \operatorname{supp}\left(\varphi_{n}\right)$. By (3.1), we have

$$
\left\langle\psi_{n}, \psi_{m}\right\rangle=0 \quad \text { and } \quad\left\langle\psi_{n},\left(H_{0}+V\right) \psi_{m}\right\rangle=0 \text { for } n \neq m
$$

Thus, by the min-max principle, $H_{0}+V$ has an infinity of eigenvalues in either $(2 \nu, \infty)$ or $(-\infty,-2 \nu)$.

Using Theorem 2.4 in place of Theorem 2.2, we get

Theorem 3.3. Let $J\left(\left\{a_{n}\right\},\left\{b_{n}\right\}\right)$ be the Jacobi matrix (1.2). Suppose $a_{n} \rightarrow 1$ and $b_{n} \rightarrow 0$ so $\sigma_{\mathrm{ess}}(J)=[-2,2]$. Let $\alpha$ be given by (2.13) and $\gamma=(2+\alpha)^{-1}$. If $J\left(\left\{a_{n}\right\},\left\{\gamma b_{n}^{2}\right\}\right)$ has at least one eigenvalue (resp., infinitely many) in $(2, \infty)$, then $J\left(\left\{a_{n}\right\},\left\{b_{n}\right\}\right)$ has at least one eigenvalue (resp., infinitely many) in $(-\infty,-2) \cup$ $(2, \infty)$.

Remark. In particular, if $J\left(\left\{a_{n}\right\},\left\{b_{n}=0\right\}\right)$ has an infinity of eigenvalues, they cannot be destroyed by a crazy choice of $\left\{b_{n}\right\}$.

\section{Essential Spectra and Compactness In Dimension 1 And 2}

Our goal in this section is to prove

Theorem 4.1. Let $\nu=1$ or 2. If $\sigma_{\mathrm{ess}}\left(H_{0}+V\right) \subset[-2 \nu, 2 \nu]$, then $V(n) \rightarrow 0$ as $|n| \rightarrow \infty$.

Theorem 4.2. If $\nu \geq 3$, there exist potentials $V$ in $\ell^{\infty}\left(\mathbb{Z}^{\nu}\right)$ so that $\sigma\left(H_{0}+V\right)=$ $[-2 \nu, 2 \nu]$ and so that $\limsup _{n \rightarrow \infty}|V(n)|>0$.

We will also provide a new proof of Theorem 2 .

The key to the dimension dependence is the issue of finding $\varphi_{n} \in \ell^{2}\left(\mathbb{Z}^{\nu}\right)$ so that $\varphi_{n}(0)=1$ and $\left\langle\varphi_{n},\left(2 \nu-H_{0}\right) \varphi_{n}\right\rangle \rightarrow 0$. We will see that this can be done in dimension 1 and 2 . It cannot be done in three or more dimensions, essentially because $\left(2 \nu-H_{0}\right)^{-1}$ exists, not as a bounded operator on $\ell^{2}$ but as a matrix defined on vectors of finite support. To minimize $\left\langle\varphi,\left(2 \nu-H_{0}\right) \varphi\right\rangle$ subject to $\varphi(0)=1$, by the method of Lagrange multipliers, one takes $\widetilde{\varphi}=\left(2 \nu-H_{0}\right)^{-1} \delta_{0} /\left\langle\delta_{0}\left(2 \nu-H_{0}\right)^{-1} \delta_{0}\right\rangle$. This is not in $\ell^{2}$ but has $\ell^{2}$ approximations. In fact, let $\varphi \in \ell^{2}$ with $\varphi(0)=\left\langle\delta_{0}, \varphi\right\rangle=$ 1. By the Cauchy-Schwarz inequality, $1 \leq\left\|\left(2 \nu-H_{0}\right)^{1 / 2} \varphi\right\|\left\|\left(2 \nu-H_{0}\right)^{-1 / 2} \delta_{0}\right\|$, that is,

$$
\left\langle\varphi,\left(2 \nu-H_{0}\right) \varphi\right\rangle \geq\left\langle\delta_{0},\left(2 \nu-H_{0}\right)^{-1} \delta_{0}\right\rangle^{-1}>0
$$

for $\nu \geq 3$. So any $\ell^{2}$ sequence $\varphi$ with $\varphi(0)=1$ has a minimal kinetic energy in dimension $\nu \geq 3$. 
A different way of thinking about this is as follows: If $\varphi$ has compact support in a box of size $L$ and $\varphi(0)=1$, then, on average, $\nabla \varphi$ is at least $L^{-1}$ so $\|\nabla \varphi\|^{2}=$ $\left\langle\varphi,\left(2 \nu-H_{0}\right) \varphi\right\rangle \sim L^{\nu} L^{-2}$. If $\nu \geq 3$, one does not do better by taking big boxes. In $\nu=1$, one certainly does; and in $\nu=2$, a careful analysis will give $(\ln L)^{-1}$ decay.

Proposition 4.3. Let $L_{1}, L_{2} \geq 1$. There exists $\varphi_{L_{1}, L_{2}} \in \ell^{2}(\mathbb{Z})$, supported in $\left[-L_{1}, L_{2}\right]$, so that

(i) $\varphi_{L_{1}, L_{2}}(0)=1$

(ii) $\left\langle\varphi_{L_{1}, L_{2}},\left(2-H_{0}\right) \varphi_{L_{1}, L_{2}}\right\rangle=\left(L_{1}+1\right)^{-1}+\left(L_{2}+1\right)^{-1}$

(iii) for suitable constants $c_{1}>0$ and $c_{2}<\infty$,

$$
c_{1}\left(L_{1}+L_{2}\right) \leq\left\|\varphi_{L_{1}, L_{2}}\right\|^{2} \leq c_{2}\left(L_{1}+L_{2}\right)
$$

Proof. Define

$$
\varphi_{L_{1}, L_{2}}(n)= \begin{cases}1-\frac{n}{L_{2}+1} & 0 \leq n \leq L_{2}+1 \\ 1-\frac{|n|}{L_{1}+1} & 0 \leq-n \leq L_{1}+1 \\ 0 & n \geq L_{2}+1 \text { or } n \leq-L_{1}-1\end{cases}
$$

then (i) and (iii) are easy. As

$$
\left\langle\psi,\left(2-H_{0}\right) \psi\right\rangle=\sum_{j=-\infty}^{\infty}[\psi(j+1)-\psi(j)]^{2}
$$

for any $\psi \in \ell^{2}(\mathbb{Z})$, we have

$$
\begin{aligned}
\left\langle\varphi_{L_{1}, L_{2}},\left(2-H_{0}\right) \varphi_{L_{1}, L_{2}}\right\rangle & =\sum_{j=1}^{L_{2}+1}\left(\frac{1}{L_{2}+1}\right)^{2}+\sum_{j=-1}^{L_{1}+1}\left(\frac{1}{L_{1}+1}\right)^{2} \\
& =\left(L_{1}+1\right)^{-1}+\left(L_{2}+1\right)^{-1}
\end{aligned}
$$

which proves (ii).

Remark. If $\psi(0)=1$ and $\psi$ is supported in $\left[-L_{1}, L_{2}\right]$,

$$
\sum_{j=1}^{L_{2}+1} \psi(j)-\psi(j-1)=-1
$$

so, by the Schwarz inequality,

$$
1 \leq\left(L_{2}+1\right) \sum_{j=1}^{L_{2}+1}|\psi(j)-\psi(j-1)|^{2}
$$

Thus

$$
\left\langle\psi,\left(2-H_{0}\right) \psi\right\rangle \geq\left(L_{1}+1\right)^{-1}+\left(L_{2}+1\right)^{-1}
$$

which shows that (4.2) is an extremal function.

Proposition 4.4. Let $L \geq 1$. There exists $\varphi_{L} \in \ell^{2}\left(\mathbb{Z}^{2}\right)$ supported in $\left\{\left(n_{1}, n_{2}\right) \mid\right.$ $\left.\left|n_{1}\right|+\left|n_{2}\right| \leq L\right\}$ so that

(i) $\varphi_{L}(0)=1$

(ii) $0 \leq\left\langle\varphi_{L},\left(4-H_{0}\right) \varphi_{L}\right\rangle \leq c[\ln (L+1)]^{-1}$ for some $c>0$

(iii) $\left(L^{-1} \ln (L)\right)^{2}\left\|\varphi_{L}\right\|^{2} \rightarrow d>0$

Remark. It seems clear that one cannot do better than $\ln (L)^{-1}$ in the large $L$ asymptotics of $\left\langle\varphi_{L},\left(4-H_{0}\right) \varphi_{L}\right\rangle$ for any test function obeying (i) and the support condition. 
Proof. Define

$$
\varphi_{L}\left(n_{1}, n_{2}\right)= \begin{cases}\frac{-\ln \left[\left(1+\left|n_{1}\right|+\left|n_{2}\right|\right) /(L+1)\right]}{\ln (L+1)} & \text { if }\left|n_{1}\right|+\left|n_{2}\right| \leq L \\ 0 & \text { if }\left|n_{1}\right|+\left|n_{2}\right| \geq L\end{cases}
$$

then (i) is obvious. As

$$
\ln \left(\frac{a+1}{(L+1)}\right)-\ln \left(\frac{a}{(L+1)}\right)=\ln \left(1+\frac{1}{a}\right) \leq a^{-1}
$$

we have that

$$
\begin{aligned}
\left\langle\varphi_{L},\left(4-H_{0}\right) \varphi_{L}\right\rangle= & \sum_{n_{1}, n_{2}}\left(\varphi_{L}\left(n_{1}+1, n_{2}\right)-\varphi_{L}\left(n_{1}, n_{2}\right)\right)^{2} \\
& +\left(\varphi_{L}\left(n_{1}, n_{2}+1\right)-\varphi_{L}\left(n_{1}, n_{2}\right)\right)^{2} \\
\leq & \ln (L+1)^{-2} \sum_{\substack{n_{1}, n_{2} \\
\left|n_{1}\right|+\left|n_{2}\right| \leq L}}\left(1+\left|n_{1}\right|+\left|n_{2}\right|\right)^{-2} \\
\leq & c \ln (L+1)^{-1}
\end{aligned}
$$

since the sum diverges as $\ln L$. This proves (ii).

To prove (iii), we note that, by a simple approximation argument,

$$
\ln (L)^{2} L^{-2}\left\|\varphi_{L}\right\|^{2} \rightarrow \iint_{|x|+|y| \leq 1}[\ln (|x|+|y|)]^{2} d x d y
$$

as $L \rightarrow \infty$.

Proof of Theorem 4.1. Consider first the case $\nu=1$. Suppose $\lim \sup |V(n)|=a>$ 0 . Pick $L$ so that $2(L+1)^{-1}<\frac{1}{8} \min \left(a^{2}, 2 a\right)$. Pick a sequence $n_{1}, \ldots, n_{j}, \ldots$ with $\left|V\left(n_{j}\right)\right| \rightarrow a$ so that $\left|n_{j}\right|-\max _{1 \leq \ell \leq j-1}\left|n_{\ell}\right| \geq 2(L+2)$. Thus, $\left|n_{j}-n_{\ell}\right| \geq 2(L+2)$ for all $j \neq \ell$.

Define

$$
F(n)=\min \left(1, \frac{2}{|V(n)|}\right)
$$

and let $\psi_{j}(n)=\varphi_{L, L}\left(n-n_{j}\right)$. Then

$$
\begin{aligned}
\left\langle\psi_{j},\left(H_{0}-2+\frac{1}{4} F V^{2}\right) \psi_{j}\right\rangle & \geq-2(L+1)^{-1}+\frac{1}{4} F\left(n_{j}\right) V\left(n_{j}\right)^{2} \\
& \geq-\frac{1}{8} \min \left(a^{2}, 2 a\right)+\frac{1}{4} \min \left(\left|V\left(n_{j}\right)\right|^{2}, 2\left|V\left(n_{j}\right)\right|\right)
\end{aligned}
$$

Thus we have that

$$
\liminf \left\langle\psi_{j},\left(H_{0}-2+\frac{1}{4} F V^{2}\right) \psi_{j}\right\rangle \geq \frac{1}{8} \min \left(a^{2}, 2 a\right)
$$

As $|F V| \leq 2$, if $\varphi_{ \pm, j}=\left(1 \pm \frac{1}{4} F V\right) \psi_{j}$, we have

$$
\frac{1}{2}\left\|\psi_{j}\right\| \leq\left\|\varphi_{ \pm, j}\right\| \leq \frac{3}{2}\left\|\psi_{j}\right\| \leq C_{L}
$$

where $C_{L}$ is independent of $j$; compare (4.1).

By (2.9), we have a subsequence of $j$ 's so that either

$$
\liminf \left\langle\varphi_{+, j_{\ell}},\left(H_{0}+V-2\right) \varphi_{+, j_{\ell}}\right\rangle \geq \frac{1}{16} \min \left(a^{2}, 2 a\right)
$$

or

$$
\liminf \left\langle\varphi_{-, j_{\ell},},\left(-H_{0}-V-2\right) \varphi_{+, j_{\ell}}\right\rangle \geq \frac{1}{16} \min \left(a^{2}, 2 a\right)
$$

Moreover, the $\varphi$ 's are orthogonal. Thus $H$ has essential spectrum in either

$$
\left[2+\frac{1}{16} d^{-1} \min \left(a^{2}, 2 a\right), \infty\right) \text { or }\left(-\infty,-2-\frac{1}{16} d^{-1} \min \left(a^{2}, 2 a\right)\right]
$$


The proof for $\nu=2$ is similar, using Proposition 4.4 in place of Proposition 4.3 .

Proof of Theorem 4.9. We will give an example with $V \geq 0$. Thus the only spectrum that $H_{0}+V$ can have outside $[-2 \nu, 2 \nu]$ is in $(2 \nu, \infty)$.

As $\nu \geq 3$, the operator $\left(2 \nu-H_{0}\right)^{-1}$ has finite matrix elements despite being unbounded. We denote the $n, m$ matrix element, the Green function, by $G_{\nu}(n-m)$. By the Birman-Schwinger principle [18, Section 3.5], if the matrix

$$
M_{n m}=V(n)^{1 / 2} G_{\nu}(n-m) V(m)^{1 / 2}
$$

defines an operator on $\ell^{2}\left(\mathbb{Z}^{\nu}\right)$ with norm strictly less than 1 , then $H_{0}+V$ has no spectrum in $(2 \nu, \infty)$.

Since $G_{\nu}(n) \rightarrow 0$ as $n \rightarrow \infty$ (indeed, it decays as $\left.|n|^{-(\nu-2)}\right)$, we can find a sequence in $\mathbb{Z}^{\nu}$ with $\left|n_{j}\right| \rightarrow \infty$ and

$$
\sum_{j \neq k}\left|G_{\nu}\left(n_{j}-n_{k}\right)\right|<\frac{1}{2}
$$

For example, pick $n_{k}$ inductively so $\sum_{j<k} G_{\nu}\left(n_{j}-n_{k}\right)<2^{-k-2}$. (Actually, $G_{\nu}(n-$ $m)>0$ for all $n$ and $m$ so the absolute value sign is redundant.) Choose $\lambda>0$ so that

and define $V$ by

$$
\lambda G_{\nu}(0)<\frac{1}{2}
$$

$$
V(n)= \begin{cases}\min (1, \lambda) & n=\text { some } n_{j} \\ 0 & \text { otherwise }\end{cases}
$$

In this way, $\lim \sup _{|n| \rightarrow \infty}|V(n)|=\min (1, \lambda)>0$. However, by Schur's lemma, $\|M\|<1$ so $H_{0}+V$ has no eigenvalues.

The ideas in the first part of this section allow us to reprove Theorem 2 and, more importantly, extend it to two dimensions.

Theorem 4.5. Let $\nu=1$ or 2 . If $\sigma\left(H_{0}+V\right) \subset[-2 \nu, 2 \nu]$, then $V=0$.

Proof. By Theorems 4.1 and 4.2, $V(n) \rightarrow 0$. By Theorem 3.1, if $H_{0}+V$ has no bound states, neither does $H_{0}+\frac{1}{4 \nu} V^{2}$. Since $V=0$ if and only if $V^{2}=0$, we may as well consider the case $V \geq 0$. Let $\varphi_{L}$ be the function guaranteed by Proposition 4.3 or 4.4. Then

$$
\left\langle\varphi_{L},\left(H_{0}+V-2 \nu\right) \varphi_{L}\right\rangle \geq V(0)+\left\langle\varphi_{L},\left(H_{0}-2 \nu\right) \varphi_{L}\right\rangle
$$

Since $\left\langle\varphi_{L},\left(H_{0}-2 \nu\right) \varphi_{L}\right\rangle \rightarrow 0$, we must have $V(0)=0$. By translation invariance, $V(n)=0$ for all $n$.

Theorem 4.6. Let $J$ be the Jacobi matrix (1.2). Suppose $\liminf a_{n} \geq 1$ and $\sigma_{\text {ess }}(J) \subset[-2,2]$. Then $b_{n} \rightarrow 0$ as $n \rightarrow \infty$.

Proof. Since $\lim \inf a_{n} \geq 1$, we can suppose $a_{n} \geq 1$ since the change from $a_{n}$ to $\min \left(a_{n}, 1\right)$ is a compact perturbation. By the lemma below, $\sigma_{\text {ess }}(J)$ can only shrink if $a_{n} \geq 1$ is replaced by $a_{n}=1$. Thus we can suppose $a_{n}=1$ in what follows.

Let $\widetilde{H}=H_{0}$ on $\ell^{2}\left(\mathbb{Z} \backslash \mathbb{Z}^{+}\right)$with a Dirichlet boundary condition at $0, \widetilde{H}=J$ on $\ell^{2}\left(\mathbb{Z}^{+}\right)$, and

$$
V(n)= \begin{cases}0 & n \leq 0 \\ b_{n} & n \geq 1\end{cases}
$$


Then $H=H_{0}+V$ differs from $\widetilde{H}$ by a finite rank perturbation. Thus $H$ has essential spectrum in $[-2,2]$. The proof is completed by using Theorem 4.1.

Lemma 4.7. If $J\left(\left\{a_{n}\right\},\left\{b_{n}\right\}\right)$ is the Jacobi matrix given by (1.2), then $\sup \sigma_{\mathrm{ess}}\left(J\left(\left\{a_{n}\right\},\left\{b_{n}\right\}\right)\right)$ and $-\inf \sigma_{\mathrm{ess}}\left(J\left(\left\{a_{n}\right\},\left\{b_{n}\right\}\right)\right)$ are monotone increasing as $a_{n}$ increases.

Proof. As noted in Section 3 of Hundertmark-Simon [6], for each $N$, the sum of the $N$ largest positive eigenvalues, $\sum_{j=1}^{N} E_{j}^{+}\left(J\left(\left\{a_{n}\right\},\left\{b_{n}\right\}\right)\right)$, is monotone in $\left\{a_{n}\right\}$. But

$$
\sup \sigma_{\mathrm{ess}}\left(J\left(\left\{a_{n}\right\},\left\{b_{n}\right\}\right)\right)=\lim _{n \rightarrow \infty} \frac{1}{N} \sum_{j=1}^{N} E_{j}^{+}\left(J\left(\left\{a_{n}\right\},\left\{b_{n}\right\}\right)\right)
$$

The proof for $-\inf \sigma_{\text {ess }}$ is similar.

\section{DECAY AND Bound States FOR HALF-Line Discrete SCHrÖDINGER Operators}

While whole-line discrete Schrödinger operators have bound states if $V \not \equiv 0$ (Theorem 2), this is not true for half-line operators. Indeed, the discrete analogue of Bargmann's bound [6] implies that

$$
\sum_{n=1}^{\infty} n|V(n)|<1 \Rightarrow \sigma\left(J_{0}+V\right)=[-2,2]
$$

where $J_{0}$ is the free Jacobi operator, that is, (1.2) with $a_{n} \equiv 1, b_{n} \equiv 0$.

One can also include the endpoint case: If a sequence of selfadjoint operators $A_{k}$ converges strongly to $A$, then

$$
\sigma(A) \subseteq \bigcap_{n} \overline{\bigcup_{k \geq n} \sigma\left(A_{k}\right)}
$$

see [13, Theorem VIII.24]. This shows that 5.1 can be extended to

$$
\sum_{n=1}^{\infty} n|V(n)| \leq 1 \Rightarrow \sigma\left(J_{0}+V\right)=[-2,2]
$$

In this section, we explore what the absence of bound states tells us about the decay of $V$. We begin with the case $V \geq 0$ :

Theorem 5.1. Suppose $V(n) \geq 0$ and that $J_{0}+V$ has no bound states. Then

$$
|V(n)| \leq n^{-1}
$$

Moreover, (5.3) cannot be improved in that for each $n_{0}$, there exists $V_{n_{0}}$ so that $V_{n_{0}}\left(n_{0}\right)=n_{0}^{-1}$ and $J_{0}+V_{n_{0}}$ has no bound states.

Proof. Let $W_{n_{0}}$ be

$$
W_{n_{0}}(n)= \begin{cases}1 & n=n_{0} \\ 0 & n \neq n_{0}\end{cases}
$$


We claim $J_{0}+\lambda W_{n_{0}}$ has a bound state if and only if $|\lambda|>n_{0}^{-1}$. By (1.6), we can suppose $\lambda>0$. In that case, by a Sturm oscillation theorem [17], there is a bound state in $(2, \infty)$ if and only if the solution of

$$
u(n+1)+u(n-1)+\lambda W_{n_{0}}(n) u(n)=2 u(n) \quad u(0)=0, u(1)=1
$$

has a negative value for some $n \in \mathbb{Z}^{+}$. The solution of (5.4) is

$$
u(n)= \begin{cases}n & n \leq n_{0} \\ n_{0}+\left(1-\lambda n_{0}\right)\left(n-n_{0}\right) & n \geq n_{0}\end{cases}
$$

which takes negative values if and only if $\lambda n_{0}>1$. This proves the claim.

In particular, $n_{0}^{-1} W_{n_{0}}=V_{n_{0}}$ is a potential where equality holds in (5.3) and $\sigma\left(J_{0}+V_{0}\right)=[-2,2]$.

On the other hand, if $V\left(n_{0}\right)>n_{0}^{-1}$, then since $V \geq 0, V(n) \geq V\left(n_{0}\right) W_{n_{0}}(n)$ for all $n$ and so, by a comparison theorem and the fact that we have shown $J_{0}+V\left(n_{0}\right) W_{n_{0}}$ has a bound state, we have that $J_{0}+V$ has a bound state. The contrapositive of $V\left(n_{0}\right)>n_{0}^{-1} \Rightarrow \sigma\left(J_{0}+V\right) \neq[-2,2]$ is the first assertion of the theorem.

Remark. Notice that Theorem 5.1 says (5.2) is optimal in the very strong sense that if $\sum_{n=1}^{\infty} \alpha_{n}|V(n)| \leq 1 \Rightarrow \sigma\left(J_{0}+V\right)=[-2,2]$ for all potentials $V$, then each $\alpha_{n} \leq n$.

Positivity of the potential made the proof of Theorem 5.1 elementary. Because of the magic of Theorem 5, we can deduce a result for $V$ 's of arbitrary sign:

Theorem 5.2. If $J_{0}+V$ has no bound states, then

$$
|V(n)| \leq 2 n^{-1 / 2}
$$

Moreover, (5.5) cannot be improved by more than a factor of 2 in that for each $n_{0}$, there exists $V_{n_{0}}$ so that $J_{0}+V_{n_{0}}$ has no bound states and

$$
\lim _{n_{0} \rightarrow \infty} n_{0}^{1 / 2}\left|V_{n_{0}}\left(n_{0}\right)\right|=1
$$

Remarks. (a) The proof shows

$$
V_{n_{0}}\left(n_{0}\right)=\sqrt{\frac{1}{n_{0}}+\frac{1}{4 n_{0}^{2}}}-\frac{1}{2 n_{0}} \equiv \beta_{n_{0}}
$$

so (5.5) cannot be improved to value better than $\beta_{n_{0}} \sim n_{0}^{-1 / 2}-\frac{1}{2} n_{0}^{-1}$.

(b) In [3] it is shown that the absence of bound states implies

$$
|V(n)| \leq \sqrt{2} n^{-1 / 2}\left(1+\frac{2}{n}\right)^{3 / 2}
$$

and that there are examples $V_{n_{0}}$ with $V_{n_{0}}\left(n_{0}\right)=\sqrt{2} n_{0}^{-1 / 2}$ and no bound states.

Proof. Theorem 5 extends to the situation where $H_{0}$ is replaced by $J_{0}$ since the mapping $\varphi \rightarrow \varphi(1 \pm F V)$ is local. Thus if $J_{0}+V$ has no bound states, neither does $J_{0}+\frac{1}{4} V^{2}$. Since $V^{2} \geq 0$, Theorem 5.1 applies, and thus $\frac{1}{4}|V(n)|^{2} \leq n^{-1}$, which is (5.5).

For the other direction, let $W_{n_{0}}$ be

$$
W_{n_{0}}= \begin{cases}1 & n=n_{0} \\ -1 & n=n_{0}+1 \\ 0 & n \neq n_{0}, n_{0}+1\end{cases}
$$


A direct solution of $(5.4)$ is

$$
u(n)= \begin{cases}n & n \leq n_{0} \\ (1-\lambda) n_{0}+1+\left(1+\lambda-\lambda^{2} n_{0}\right)\left(n-n_{0}-1\right) & n \geq n_{0}+1\end{cases}
$$

Thus $u(n)$ has a negative value if and only if $1+\lambda-\lambda^{2} n_{0}<0$. Define

$$
\lambda_{ \pm}^{\text {crit }}= \pm \sqrt{\frac{1}{4 n_{0}^{2}}+\frac{1}{n_{0}}}-\frac{1}{2 n_{0}}
$$

If $|\lambda|>\min \left(\left|\lambda_{+}^{\text {crit }}\right|,\left|\lambda_{-}^{\text {crit }}\right|\right), u$ takes negative values for either $u(n, \lambda)$ or $u(n,-\lambda)$. By (1.6), $J_{0}+V$ has eigenvalues in $(-\infty,-2)$ if and only if $J_{0}-V$ has eigenvalues in $(2, \infty)$. Thus since $\left|\lambda_{+}^{\text {crit }}\right|<\left|\lambda_{-}^{\text {crit }}\right|, J_{0}+\lambda W_{n_{0}}$ has no eigenvalues if $|\lambda| \leq \lambda_{+}^{\text {crit }}$.

One can also say something about infinitely many bound states:

Theorem 5.3. (i) If $V \geq 0$ and

$$
\limsup _{n \rightarrow \infty}|V(n)| n>1
$$

then $J_{0}+V$ has infinitely many bound states.

(ii) For general $V$, if $\limsup _{n \rightarrow \infty}|V(n)| n^{1 / 2}>2$, then $J_{0}+V$ has infinitely many bound states.

Proof. (ii) follows from (i) by Theorem 5. To prove (i), suppose $J_{0}+V$ has only finitely many bound states. Then $\left(J_{0}+V-2\right) u$ has only finitely many sign changes, so there is $N_{0}$ with $u(n) u(n+1)>0$ if $n>N_{0}$. It follows that $J_{0}+V$ with $\widetilde{V}(n)=$ $V\left(n+N_{0}\right)$ has no bound states. Thus $|\widetilde{V}(n)| \leq n^{-1}$, so $\lim _{\sup _{n \rightarrow \infty}} n|V(n)| \leq 1$. Thus, by contrapositives, (5.8) implies $J_{0}+V$ has infinitely many bound states.

Example 5.4. Let $N$ be a positive integer and $n_{k}=N^{2 k}$. We consider the sequence $u(n)$ which has slope $u(n+1)-u(n)=N^{-k}$ for $n \in\left[n_{k}, n_{k+1}\right)$ and then determine the potential $V$ at the sites $n_{k}$ so that $u$ is the generalized eigenfunction at energy 2. (Constancy of the slope in the intervals $\left(n_{k}, n_{k+1}\right)$ implies that the potential vanishes there.) We have

$$
\begin{aligned}
u\left(n_{k}\right) & =n_{1}+\left(n_{2}-n_{1}\right) N^{-1}+\cdots+\left(n_{k}-n_{k-1}\right) N^{-(k-1)} \\
& =\left(1-N^{-1}\right)\left\{N^{2}+N^{3}+\cdots+N^{k}\right\}+N^{k+1} \\
& =N^{k+1}\left\{1+N^{-1}-N^{-k}\right\}
\end{aligned}
$$

and so

$$
\begin{aligned}
V\left(n_{k}\right) & =\frac{2 u\left(n_{k}\right)-u\left(n_{k}+1\right)-u\left(n_{k}-1\right)}{u\left(n_{k}\right)} \\
& =\frac{N^{1-k}-N^{-k}}{N^{k+1}\left\{1+N^{-1}-N^{-k}\right\}} \\
& =\frac{1-N^{-1}}{1+N^{-1}-N^{-k}} \frac{1}{n_{k}}
\end{aligned}
$$

As $u$ is monotone, there are no sign flips. We may conclude that $J_{0}+V$ has no bound states because $V(n) \geq 0$. Therefore, taking $N \rightarrow \infty$, we see that the 1 in (5.8) is optimal.

A similar argument 19] shows there are examples with $\lim \sup n^{1 / 2}|V(n)|=1-\varepsilon$ and no bound states for each $\varepsilon>0$. Basically, $V(n) \neq 0$ for $n=n_{k}$ or $n_{k}+1$ and 
$V\left(n_{k}\right)=-V\left(n_{k}+1\right)=n_{k}^{-1 / 2}\left(1-\varepsilon_{k}\right)$ with $\varepsilon_{k} \rightarrow \varepsilon$. Again, $n_{k}$ must grow at least geometrically.

The examples that saturate Theorems 5.1 and 5.3 are sparse, that is, mainly zero. If $V$ is mainly nonzero and comparable in size, the borderlines change from $n^{-1}$ to $n^{-2}$ for positive $V$ 's and from $n^{-1 / 2}$ to $n^{-1}$ for $V$ 's of arbitrary sign.

Theorem 5.5. Let $V \geq 0$. Suppose there exists $\varepsilon>0$ and $n_{k} \rightarrow \infty$ so that (i)

$$
\frac{2}{n_{k}} \sum_{j=n_{k} / 2}^{n_{k}} V(j) \geq \varepsilon V\left(n_{k}\right)
$$

(ii) $\lim \sup _{k \rightarrow \infty} \varepsilon n_{k}^{2} V\left(n_{k}\right)>48$

Then $J_{0}+V$ has infinitely many bound states.

Proof. For notational simplicity, we suppose each $n_{k}$ is a multiple of 4 . By passing to a subsequence, we can suppose that

$$
\begin{gathered}
\frac{\varepsilon n_{k}}{8} V\left(n_{k}\right)>\frac{6}{n_{k}} \\
\frac{n_{k+1}}{4}>\frac{3}{2} n_{k}+2
\end{gathered}
$$

Let $u_{k}$ be the function which is 1 at $n_{k}$, has constant slope on the intervals $\left[\frac{n_{k}}{4}-1, n_{k}\right]$ and $\left[n_{k}, \frac{3 n_{k}}{2}+1\right]$, and vanishes at $n=\frac{n_{k}}{4}-1$ and $n=\frac{3 n_{k}}{2}+1$. By Proposition 4.3,

$$
\left\langle u_{k},\left(2-J_{0}\right) u_{k}\right\rangle \leq \frac{6}{n_{k}}
$$

On $\left[\frac{n_{k}}{2}, n_{k}\right]$, we have $|u(j)| \geq \frac{1}{2}$, so

$$
\left\langle u_{k}, V u_{k}\right\rangle \geq \frac{1}{4} \sum_{j=n / 2}^{n} V(j) \geq \frac{\varepsilon n_{k}}{8} V\left(n_{k}\right) \quad \text { (by (5.9)) }
$$

By 5.10$),\left\langle u_{k},\left(J_{0}+V-2\right) u_{k}\right\rangle>0$ for all $k$. By (5.11) for $k \neq \ell$,

$$
\left\langle u_{k}, u_{\ell}\right\rangle=\left\langle u_{k},\left(J_{0}+V\right) u_{\ell}\right\rangle=0
$$

so, by the min-max principle, $J_{0}+V$ has infinitely many eigenvalues in $(2, \infty)$.

Theorem 5 and Theorem 5.5 immediately imply

Theorem 5.6. Suppose there exists $\varepsilon>0$ and $n_{k} \rightarrow \infty$ so that

(i) $\frac{2}{n_{k}} \sum_{j=n_{k} / 2}^{n_{k}}|V(j)|^{2} \geq \varepsilon^{2}\left|V\left(n_{k}\right)\right|^{2}$

(ii) $\limsup _{k \rightarrow \infty} \varepsilon n_{k}\left|V\left(n_{k}\right)\right|>8 \sqrt{3}$

Then $J_{0}+V$ has infinitely many bound states.

In this regard, here is another application of Theorem 5:

Theorem 5.7. If $|V(n)| \geq \frac{\beta}{n}$ with $\beta>1$ and $V(n) \rightarrow 0$, then $J_{0}+V$ has infinitely many bound states.

Proof. It is known (see [2, Theorem A.7]) if $\beta^{2}>1$, then the operator with potential $\frac{\beta^{2}}{4 n^{2}}$, and hence the operator with potential $\frac{1}{4} V(n)^{2} \geq \frac{\beta^{2}}{4 n^{2}}$, has infinitely many bound states. The assertion now follows from Theorem 5 . 
Corollary 5.8. If $V(n) \rightarrow 0$ but $\liminf _{|n| \rightarrow \infty}|n V(n)|>1$, then $J_{0}+V$ has infinitely many bound states. The same result holds in the whole-line setting.

Proof. We begin with the half-line case. By hypothesis, there exists a $\beta>1$ such that $|V(n)| \geq \frac{\beta}{n}$ for all but finitely many $n$. Therefore the claim follows from the previous theorem because a finite rank perturbation can remove at most finitely many eigenvalues. The whole-line case follows by Dirichlet decoupling.

Remark. It is known (see [2]) that if $V(n)=\frac{1}{4 n^{2}}$ or $V(n)=\beta \frac{(-1)^{n}}{n}$ with $|\beta|<\frac{1}{2}$, then $J_{0}+V$ has finitely many bound states. Thus the powers $n^{-2}$ and $n^{-1}$ in the previous results are optimal.

The optimal constant in Theorem 5.7 is 1 , as we now show.

Proposition 5.9. For $\beta \in[-1,1]$, the operator $J_{0}+V$ with potential $V(n)=$ $\beta \frac{(-1)^{n}}{n}$ has no bound states.

Proof. We will show that the operator with potential $V(n)=\frac{(-1)^{n}}{n}$ has no bound states. As the absolute value of a bound state eigenvalue is an increasing function of the coupling constant, this implies that potentials of the form $V(n)=\beta \frac{(-1)^{n}}{n}$ have no bound states for $\beta \in[0,1]$. Equation (2.5) shows that $J_{0}+V$ is unitarily equivalent to $-\left(J_{0}-V\right)$. Thus, the proposition for $\beta \in[-1,0]$ follows from the $\beta \in[0,1]$ case.

By the unitary equivalence of $J_{0}+V$ and $-\left(J_{0}-V\right)$, it suffices to show that for $V_{0}=(-1)^{n} / n, J_{0}+V_{0}$ and $J_{0}-V_{0}$ have no eigenvalues in $(2, \infty)$.

We look at solutions of

$$
u(n+1)+u(n-1)=\left(2 \mp V_{0}(n)\right) u(n)
$$

By Sturm oscillation theory, the number of eigenvalues of $J_{0} \pm V_{0}$ in $(2, \infty)$ is equal to the number of zeros, in $(0, \infty)$, of the linear interpolation of the generalized eigenfunction - that is, the solution of (5.12) with $u(0)=0$. Moreover, the Sturm separation theorem implies that if (5.12) has a solution with $u(n)>0$ for $n=$ $0,1,2, \ldots$, then the generalized eigenfunction must be positive for $n \geq 1$ (and not $\ell^{2}$; see remark below).

We are able to write down positive solutions explicitly, but rather than pull such a rabbit out of a hat, we provide some explanation. Motivated by calculations in Maple, we look for solutions with $u(n)=u(n+1)$ for either all odd $n$ or all even $n$. This is equivalent to asking if

$$
\left(\begin{array}{rr}
x & -1 \\
1 & 0
\end{array}\right)\left(\begin{array}{rr}
y & -1 \\
1 & 0
\end{array}\right)=\left(\begin{array}{cc}
x y-1 & -x \\
y & -1
\end{array}\right)
$$

has $\left(\begin{array}{l}1 \\ 1\end{array}\right)$ as an eigenvector. If this is true for $y=E-V(n), x=E-V(n+1)$ for all odd (resp. even) $n$, then the Schrödinger equation has a solution with $u(n)=u(n-1)$ for all odd (resp. even) $n$, and for such $n$,

$$
u(n+2)=[E-V(n)-1] u(n)
$$

The matrix in (5.13) has $\left(\begin{array}{l}1 \\ 1\end{array}\right)$ as an eigenvector if and only if

$$
x y=x+y
$$

If $x=2+a, y=2+b$, then (5.12) becomes

$$
a b=-a-b
$$


This is solved by $b=\frac{1}{m}, a=-\frac{1}{m+1}$ with $y-1=1+\frac{1}{m}$. Since $-V(n)$ appears in the transfer matrix for $V_{0}$, we take $m=2 n+1, n=0,1,2, \ldots$ and find a solution with

$$
u(0)=u(1)=1 \quad u(2 n)=u(2 n+1), \quad u(2 n+2)=\left(1+\frac{1}{2 n+1}\right) u(2 n)
$$

which is a positive solution with $u(n) \rightarrow \infty$ as $n^{1 / 2}$ as $n \rightarrow \infty$. For $-V_{0}$, we take $m=2 n, n=1,2, \ldots$, and find a solution with

$$
u(0)=0 \quad u(1)=u(2)=1 \quad u(2 n)=u(2 n-1) \quad u(2 n+2)=\left(1+\frac{1}{2 n}\right) u(2 n)
$$

so again, $u(n) \rightarrow \infty$ as $n^{1 / 2}$. We have thus found the required solution to show $J_{0}+V_{0}$ has no eigenvalues in $(2, \infty)$.

Remarks. (a) It follows from the proof that the generalized eigenfunctions at energies \pm 2 are not square summable. This shows that \pm 2 are not eigenvalues.

(b) Choosing $y=-\frac{1}{m}, x=\frac{1}{m+1}$ in the arguments given above shows that there are solutions $u_{ \pm}$of $\left(J_{0}+V_{0}\right) u=0$ with $\left|u_{ \pm}(n)\right| \sim|n|^{ \pm 1 / 2}$ as $n \rightarrow \infty$. This shows that 0 is not an eigenvalue of $J_{0}+V_{0}$ but suggesting that for $J_{0}+(1+\varepsilon) V_{0}$, there are solutions $\ell^{2}$ at infinity for $\varepsilon>0$. That is, just as coupling 1 is the borderline for eigenvalues outside $[-2,2]$, it is the borderline for an eigenvalue at $E=0$ similar to the Wigner-von Neumann phenomenon.

As our final topic, we want to discuss divergence of eigenvalue moments if $|V(n)| \sim n^{-\alpha}$ with $\alpha<1$.

Lemma 5.10. Let $A$ be a bounded selfadjoint operator. Let $\left\{\varphi_{j}\right\}_{j=1}^{\infty}$ be an orthonormal set with

$$
\left\langle\varphi_{j}, A \varphi_{k}\right\rangle=\alpha_{j} \delta_{j k}
$$

If $F$ is a nonnegative even function on $\mathbb{R}$ that is monotone nondecreasing on $[0, \infty)$, then

$$
\operatorname{Tr}(F(A)) \geq \sum_{j} F\left(\alpha_{j}\right)
$$

Remarks. (a) As $F(A) \geq 0$, it follows that $\operatorname{Tr}(F(A))$ is always defined although it may be infinite.

(b) In particular, if $\varphi_{j}$ is a family of nonzero vectors in $\ell^{2}\left(\mathbb{Z}^{+}\right)$with $\operatorname{dist}\left(\operatorname{supp}\left(\varphi_{j}\right), \operatorname{supp}\left(\varphi_{k}\right)\right) \geq 2$ for $j \neq k$, then for $J=J_{0}+V$,

$$
\operatorname{Tr}(F(J)) \geq \sum_{j} F\left(\left|\frac{\left\langle\varphi_{j}, J \varphi_{j}\right\rangle}{\left\langle\varphi_{j}, \varphi_{j}\right\rangle}\right|\right)
$$

Proof. Let $E_{1} \geq E_{2} \geq \cdots$ be the eigenvalues of $|A|$. By min-max and max-min for $A$, we have $E_{j} \geq\left|\alpha_{j}\right|^{*}$ where $\left|\alpha_{j}\right|^{*}$ is the decreasing rearrangement of $\left|\alpha_{j}\right|$. So (5.18) follows.

Lemma 5.11. Let $|V| \leq 4 \nu$ on $\operatorname{supp}(\varphi)$. Then there exists $\psi$ with $\operatorname{supp}(\psi)=$ $\operatorname{supp}(\varphi)$ so that

$$
\|\psi\|^{-2}\left|\left\langle\psi,\left(H_{0}+V\right) \psi\right\rangle\right|-2 \nu \geq \frac{1}{4}\left[\|\varphi\|^{-2}\left\langle\varphi,\left(H_{0}+\frac{1}{4 \nu} V^{2}\right) \varphi\right\rangle-2 \nu\right]
$$

Proof. Let $\psi_{ \pm}=\left(1 \pm(4 \nu)^{-1} V\right) \varphi$. Since $|V| \leq 4 \nu,\left\|\psi_{ \pm}\right\|^{2} \leq 4\|\varphi\|^{2}$. The result now follows from (2.9) by choosing $\psi$ to be either $\psi_{+}$or $U \psi_{-}$. 
Theorem 5.12. Let $J$ be a Jacobi matrix of the form $J_{0}+V$ where

$$
|V(n)| \geq C n^{-\alpha}
$$

for some $\alpha<1$ and $V(n) \rightarrow 0$. Then

$$
\sum_{j}\left(\left|E_{j}\right|-2\right)^{\gamma}=\infty
$$

for

$$
\gamma<\frac{1-\alpha}{2 \alpha}
$$

where $E_{j}$ are eigenvalues of $J$ outside $[-2,2]$.

Remark. In particular, the eigenvalue sum $\sum_{j=1}^{\infty}\left(\left|E_{j}\right|-2\right)^{1 / 2}$ critical of Szegötype sum rules [7, 16] diverges if $\alpha<\frac{1}{2}$. This illuminates results in [2, 16.

Proof. Fix $p>0$. Let $\varphi_{m}$ be supported near $m^{p+1}$ on an interval $\left[m^{p+1}-\right.$ $\left.C_{1} m^{p}, m^{p+1}+C_{1} m^{p}\right]$ where $C_{1}$ is picked to arrange that supports are separated by at least 2. Taking the slopes fixed on each half-interval and using Proposition 4.3. we see

$$
\begin{aligned}
\left\langle\varphi_{m},\left(2-H_{0}\right) \varphi_{m}\right\rangle & \leq \frac{C_{2}}{m^{p}} \\
\left\langle\varphi_{m}, \frac{1}{4} V^{2} \varphi_{m}\right\rangle & \geq \frac{C_{3} m^{p}}{m^{2 \alpha(p+1)}} \\
\left\langle\varphi_{m}, \varphi_{m}\right\rangle & \geq C_{4} m^{p}
\end{aligned}
$$

So long as $\alpha(p+1)<p$ (i.e., $\left.p<\frac{\alpha}{1-\alpha}\right)$, (5.25) beats out (5.24) for large $m$, and we find

$$
\left\langle\varphi_{m}, \varphi_{m}\right\rangle^{-1}\left\langle\varphi_{m},\left(H_{0}+\frac{1}{4} V^{2}-2\right) \varphi_{m}\right\rangle \geq C_{5} m^{-2 \alpha(p+1)}
$$

As $p \downarrow \frac{\alpha}{1-\alpha}, 2 \alpha(p+1) \downarrow \frac{2 \alpha}{1-\alpha}$.

By the lemma with $F(x)=\operatorname{dist}(x,[-2,2])^{\gamma}$, we see that we have divergence if (5.23) holds.

Remarks. (a) If the constant $C$ in (5.21) is large enough, we can take $p=\frac{\alpha}{1-\alpha}$ and get divergence if $\gamma=\frac{1-\alpha}{2 \alpha}$.

(b) One can extend this result as well as Theorems 5.3 and 5.5 to higher dimensions.

\section{REFERENCES}

[1] M. Cwikel, Weak type estimates for singular values and the number of bound states of Schrödinger operators, Ann. of Math. 106 (1977), 93-100.

[2] D. Damanik, D. Hundertmark, and B. Simon, Bound states and the Szegö condition for Jacobi matrices and Schrödinger operators, preprint.

[3] D. Damanik and R. Killip, in preparation.

[4] P. Deift and B. Simon, Almost periodic Schrödinger operators, III. The absolutely continuous spectrum in one dimension, Comm. Math. Phys. 90 (1983), 389-411.

[5] S. Denisov, On Nevai's conjecture and Rakhmanov's theorem for Jacobi matrices, preprint

[6] D. Hundertmark and B. Simon, Lieb-Thirring inequalities for Jacobi matrices, J. Approx. Theory 118 (2002), 106-130.

[7] R. Killip and B. Simon, Sum rules for Jacobi matrices and their applications to spectral theory, to appear in Ann. of Math. 
[8] M. Klaus, On the bound state of Schrödinger operators in one dimension, Ann. Phys. 108 (1977), 288-300.

[9] L.D. Landau and E.M. Lifshitz, Quantum Mechanics: Non-relativistic Theory. Course of Theoretical Physics, Vol. 3, Addison-Wesley, Reading, Mass., 1958.

[10] E.H. Lieb, Bounds on the eigenvalues of the Laplace and Schrödinger operators, Bull. Amer. Math. Soc. 82 (1976), 751-753; see also The number of bound states of one-body Schrödinger operators and the Weyl problem, in "Geometry of the Laplace Operator (Proc. Sympos. Pure Math., Univ. Hawaii, Honolulu, Hawaii, 1979), pp. 241-252, Proc. Sympos. Pure Math., XXXVI, Amer. Math. Soc., Providence, R.I., 1980.

[11] P. Nevai, Weakly convergent sequences of functions and orthogonal polynomials, J. Approx. Theory 65 (1991), 322-340.

[12] E.A. Rakhmanov, On the asymptotics of the ratio of orthogonal polynomials, II, Math. USSR Sb. 46 (1983), 105-117.

[13] M. Reed and B. Simon, Methods of Modern Mathematical Physics. I. Functional Analysis, Academic Press, New York, 1980.

[14] G.V. Rozenblum, Distribution of the discrete spectrum of singular differential operators, Dokl. Akad. Nauk SSSR 202 (1972), 1012-1015); Izv. VUZaved. Matematika 1 (1976), 75-86. [Russian]

[15] B. Simon, The bound state of weakly coupled Schrödinger operators in one and two dimensions, Ann. Phys. 97 (1976), 279-288.

[16] B. Simon and A. Zlatoš, Sum rules and the Szegö condition for orthogonal polynomials on the real line, preprint

[17] G. Teschl, Jacobi Operators and Completely Integrable Nonlinear Lattices, Mathematical Surveys and Monographs Vol. 72, American Mathematical Society, Providence, R.I., 2000.

[18] W. Thirring, A Course in Mathematical Physics. Vol. 3. Quantum Mechanics of Atoms and Molecules, Lecture Notes in Physics, 141. Springer-Verlag, New York-Vienna, 1981.

[19] A. Zlatoš, private communication. 